\title{
The Ernst equation on a Riemann surface
}

\author{
D. Korotkin ${ }^{1}$, H. Nicolai \\ II. Institute for Theoretical Physics. Hamburg University, Luruper Chaussee 149, Hamburg 22761, Germany
}

Received 17 May 1994; accepted 20 June 1994

\begin{abstract}
The Emst equation is formulated on an arbitrary Riemann surface. Analytically, the problem reduces to finding solutions of the ordinary Ernst equation which are periodic along the symmetry axis. The family of (punctured) Riemann surfaces admitting a non-trivial Emst field constitutes a "partially discretized" subspace of the usual moduli space. The method allows us to construct new exact solutions of Einstein's equations in vacuo with non-trivial topology, such that different "universes", each of which may have several black holes on its symmetry axis, are connected through necks bounded by cosmic strings. We show how the extra topological degrees of freedom may lead to an extension of the Geroch group and discuss possible applications to string theory.
\end{abstract}

\section{Introduction}

General investigations of axisymmetric stationary solutions of Einstein's equations in vacuo are commonly based on the Ernst equation [1,2]

$$
(\mathcal{E}+\overline{\mathcal{E}})\left(\mathcal{E}_{\bar{z} \bar{z}}-\frac{\rho_{\bar{z}} \mathcal{E}_{\bar{z}}+\rho_{\bar{z}} \mathcal{E}_{z}}{2 \rho}\right)=2 \mathcal{E}_{z} \mathcal{E}_{\bar{z}}
$$

which constitutes the main part of Einstein's equations. Here, the complex variable $z=u+i v$ parametrizes the two (space-like) coordinates, on which the complex Ernst potential $\mathcal{E}(z, \bar{z})$, and hence the metric depend. The real function $\rho(z, \bar{z})$ (related to the 33-component of the 4-metric) is harmonic, viz.

$$
\rho_{z \bar{z}}=0
$$

\footnotetext{
${ }^{1}$ Supported by Alexander von Humboldt Foundation.

0550-3213/94/\$07.00 (C) 1994 Elsevier Science B.V. All rights reserved SSDI 0550-3213(94)00284-L
} 
and can thus be represented as the imaginary part of some (locally) holomorphic function $\xi(z)$, i.e.

$$
\rho(z, \bar{z})=\operatorname{Im} \xi(z) .
$$

Defining

$$
x(z, \bar{z})=\operatorname{Re} \xi(z)
$$

we can rewrite (1) as follows:

$$
(\mathcal{E}+\overline{\mathcal{E}})\left(\mathcal{E}_{x x}+\frac{1}{\rho} \mathcal{E}_{\rho}+\mathcal{E}_{\rho \rho}\right)=2\left(\mathcal{E}_{x}^{2}+\mathcal{E}_{\rho}^{2}\right),
$$

or, in complex notation,

$$
(\mathcal{E}+\overline{\mathcal{E}})\left(\mathcal{E}_{\xi \bar{\xi}}-\frac{\mathcal{E}_{\xi}-\mathcal{E}_{\bar{\xi}}}{2(\xi-\bar{\xi})}\right)=2 \mathcal{E}_{\xi} \mathcal{E}_{\bar{\xi}}
$$

At this point, one usually switches from the coordinates $(z, \bar{z})$ to new coordinates $(x, \rho)$ (so-called Weyl canonical coordinates) by means of the conformal reparametrization $z \rightarrow \xi(z)$. The full metric of the Einstein manifold $\mathcal{M}$ corresponding to a given solution of (1) can then be represented in the form

$$
\mathrm{d} s^{2}=\mathrm{d} l^{2}+\mathrm{d} L^{2}
$$

where

$$
\begin{aligned}
\mathrm{d} l^{2} & =f^{-1} \mathrm{e}^{2 k} \mathrm{~d} \xi \mathrm{d} \bar{\xi}, \\
\mathrm{d} L^{2} & =-f(\mathrm{~d} t+A \mathrm{~d} \phi)^{2}+f^{-1} \rho^{2} \mathrm{~d} \phi^{2} .
\end{aligned}
$$

The variables $t$ and $\phi$ are the time and angular coordinates, respectively, so that there are two mutually commuting Killing vectors $\partial_{t}$ and $\partial_{\phi}$, one time-like and one space-like. The functions $f, A$ and $k$ depending on $(\xi, \bar{\xi})$ are determined from

$$
f=\operatorname{Re} \mathcal{E}, \quad A_{\xi}=2 \rho \frac{(\mathcal{E}-\overline{\mathcal{E}})_{\xi}}{(\mathcal{E}+\overline{\mathcal{E}})^{2}}, \quad k_{\xi}=2 i \rho \frac{\mathcal{E}_{\xi} \overline{\mathcal{E}}_{\xi}}{(\mathcal{E}+\overline{\mathcal{E}})^{2}} .
$$

Observe that the first order differential equations can be consistently solved because the compatibility with the complex conjugate equations is guaranteed by $(5)$.

Although the change of variables from $(z, \bar{z})$ to $(x, \rho)$ is purely local, a simple global topology has been assumed in all previous studies of axisymmetric stationary solutions of Einstein's equations [2]. Namely, one takes the complex coordinate $x+i \rho$ to parametrize the upper half plane, such that $x \in \mathbb{R}$ parametrizes the symmetry axis and $\rho \geqslant 0$ is a radial variable. The class of exact solutions obtained in this way includes the well known Schwarzschild and Kerr solutions, which provide the mathematical background for black hole physics, as well as a host of other solutions with unphysical features. In this paper, we want to exploit the link between (1) and (5) at the global level by assuming $(z, \bar{z})$ to be local coordinates on an arbitrary Riemann surface rather 
than (part of) the Riemann sphere. The function $\xi(z)$ is then no longer globally singlevalued. An important element in our construction is the Kaluza-Klein type interpretation of the metric (6) as a model of matter-coupled gravity in two dimensions. Then the part $\mathrm{d} l^{2}$ of the metric (6) is interpreted as the metric of a two-dimensional world-sheet with local coordinates $(z, \bar{z})$, while the Ernst potential $\mathcal{E}$ is regarded as a complex "matter field" living on this world-sheet together with the "dilaton" field $\rho$ and the Liouville degree of freedom contained in $k(z, \bar{z})$. On the basis of this interpretation, we will construct new exact solutions of Einstein's equations topologically equivalent to the product of the time axis (parametrized by $t$ ), the circle $S^{1}$ (parametrized by $\phi$ ) and (part of) some Riemann surface $\mathcal{L}$. Even for the trivial solution $\mathcal{E}=1$, we obtain topologically non-trivial flat manifolds with an arbitrary number of asymptotically flat regions. To be sure, such manifolds will have singularities of some kind. As it turns out, the singularities of the four-manifold correspond to $\delta$-function curvature singularities on the world-sheet. In the 4D interpretation, these can be viewed as ringlike cosmic strings with negative tension (i.e. instead of a deficit angle in the neighborhood of the string, there is now an excess angle). We believe that the solutions presented here may have some physical interest since they provide novel examples of wormhole-type solutions for Einstein's equations without matter.

To define the Ernst potential $\mathcal{E}$ on the Riemann surface $\mathcal{L}$ and to exploit the global freedom left in the choice of $\xi(z)$, we utilize a description of $\mathcal{L}$ introduced by Mandelstam [3] and further elaborated in Ref. [4] for the computation of multiple string scattering in the light-cone gauge. To this aim, we choose a meromorphic abelian differential $\mathrm{d} \xi(P)$ on $\mathcal{L}$ (points on $\mathcal{L}$ are labeled by $P, Q, \ldots$ ) and define

$$
\xi(P)=\int_{P_{0}}^{P} \mathrm{~d} \xi,
$$

where the base point $P_{0} \in \mathcal{L}$ must not coincide with any singular point of $\mathrm{d} \xi(P)$, but otherwise can be chosen arbitrarily. The real and imaginary parts of $\xi(P)$ will be designated by $x(P)$ and $\rho(P)$ as in (3) and (4), respectively. The differential $\mathrm{d} \xi(P)$ defines a flat metric on $\mathcal{L}$ through

$$
\mathrm{d} s_{0}^{2}=\mathrm{d} \xi \otimes \mathrm{d} \bar{\xi} .
$$

Obviously, $\mathrm{d} s_{0}^{2}$ is degenerate at the zeros and singular at the poles of $\mathrm{d} \xi(P)$. Moreover, the function $\xi(P)$ is in general not globally defined on $\mathcal{L}$ since the corresponding abelian integral generically has non-zero cyclic periods. If we want to define the Ernst potential $\mathcal{E}=\mathcal{E}(x(P), \rho(P))$ on $\mathcal{L}$ by making use of the local equivalence of $(1)$ and (5), we must impose additional conditions on $\mathcal{L}$ and the Ernst potential in order to render $\mathcal{E}(x, \rho)$ globally single-valued. We will need two requirements.

(i) While the cyclic periods of the differential $\mathrm{d} \xi(P)$ do not vanish in general, we can always arrange them to be real by the addition of suitable holomorphic differentials (this defines $\mathrm{d} \xi$ uniquely). Consequently, the function $\rho(P)=\operatorname{Im} \xi(P)$ is globally defined on $\mathcal{L}$; it plays the role of a global (light-cone) time in the Mandelstam 
description of string scattering. The single-valuedness of $\rho$ is also indispensable for single-valuedness of the corresponding metric in four dimensions.

(ii) If the function $x(P)=\operatorname{Re} \xi(P)$ is not globally defined, the Ernst potential must be periodic in its first argument with a certain period $L$, i.e. $\mathcal{E}(x, \rho)=\mathcal{E}(x+L, \rho)$. In addition we must assume the (real) cyclic periods $C_{j}$ of the integral $\xi(P)$ to be "quantized", i.e. $C_{j}=n_{j} L$ where $n_{j} \in \mathbb{Z}$ and $L \in \mathbb{R}$. Under these conditions $\mathcal{E}$ extends to a globally single-valued function on the surface $\mathcal{L}$.

In the special case when all cyclic periods of $\mathrm{d} \xi(P)$ vanish, we can drop the second requirement since then $\mathrm{d} \xi(P)=\mathrm{d} h(P)$ for some meromorphic function $h(P)$ on $\mathcal{L}$. Periodicity for $\mathcal{E}(x, \rho)$ is not required in this case, and for any solution $\mathcal{E}(\xi, \bar{\xi})$ of (5) we get a non-trivial and globally defined Ernst potential $\mathcal{E}(h(z), \bar{h}(z))$ on $\mathcal{L}$. The topological degrees of freedom can be analyzed by switching off the "matter excitations", i.e. by putting $\mathcal{E}=1$; they are described by the ordinary moduli space of the associated (punctured) Riemann surface ${ }^{2}$. If we now switch on some non-trivial solution of (5) with period $L$, the quantization condition above will lead to a (partial) discretization of moduli space since all cyclic periods of the integral $\mathrm{d} \xi$ are proportional to $L$ with integer coefficients. Thus the Riemann surfaces amenable to our construction constitute a "partially discretized" subspace of ordinary moduli space. On the world-sheet $\mathcal{L}$, we can get additional branch points by choosing $\mathcal{E}(\xi, \bar{\xi})$ and/or $f^{-1} \mathrm{e}^{2 k}$ with extra branch points in the $\xi$-plane.

The existence of topologically non-trivial solutions has important implications for the symmetry structure of the theory. As has been known for a long time, the Ernst equation belongs to the class of integrable equations. The first indication of this basic property appeared in Ref. [5] where it was shown that the Ernst equation admits an infinite-dimensional symmetry group (Geroch group) acting on the space of axisymmetric stationary solutions. The integrability of Einstein's equations in this reduction was subsequently demonstrated in Ref. [6] and Ref. [7], where the associated zero curvature representation (or Lax pair) was found. The application of the methods of soliton theory by many authors allowed the construction of multi-soliton solutions [6] and a study of the associated Riemann-Hilbert problem [8,9]. Furthermore, the related Bäcklund transformations were found in Ref. [10], and a link between the Ernst equation and the deformation of hyperelliptic algebraic curves was established in Ref. [11]. The Kerr solution was interpreted in this framework as a special case of the two-soliton metric. It should be kept in mind, however, that most of the new solutions generated by this method are plagued by unphysical features such as naked singularities and violations of causality [12-14] (this statement applies to both stationary axisymmetric and colliding plane wave solutions; see e.g. Refs. $[15,16]$ for reviews of the latter ${ }^{3}$ ). The understanding of the underlying group theoretical structure evolved from the early discovery of a "hidden" $S L(2, \mathbb{R})$ symmetry in Einstein's equations to detailed studies

\footnotetext{
${ }^{2}$ The full "moduli space of solutions" corresponding to the gauge equivalence classes of solutions in the presence of matter is, of course, infinite-dimensional.

${ }^{3}$ The colliding plane wave solutions can be formally obtained from the axisymmetric stationary ones by a Wick-rotation (although their physical interpretation is, of course, entirely different). In terms of the notation adopted in Ref. [16], this amounts to the replacement of $z, \bar{z}, \xi(z)$ and $\bar{\xi}$ by by $x^{+}, x^{-}, \rho_{+}\left(x^{+}\right)$and $\rho_{-}\left(x^{-}\right)$, respectively.
} 
of the infinite solution generating symmetries of the axisymmetric reduction [17]. It advanced considerably with the advent of (Kaluza-Klein) supergravity and the discovery of hidden symmetries in these theories [18], which made it abundantly clear that Einstein's theory is just a special example in a more general class of (possibly supersymmetric) $G / H$ coset space sigma models coupled to gravity. This led to the realization that the Geroch group is nothing but (a non-linear realization of) the loop group $S \widehat{L(2, \mathbb{R})}$ with a central extension acting as a scaling operator on the conformal factor and that the emergence of affine Kac-Moody algebras in the reduction to two dimensions is a general phenomenon $[19,20,16]$; in fact, even the $G / H$ coset structure of the higher-dimensional theories has an infinite-dimensional counterpart. On the basis of earlier conjectures that these affine Kac-Moody algebras admit hyperbolic extensions in the reduction to one dimension [21], it was suggested in Ref. [22] that there should exist new symmetries associated with the topological world-sheet degrees of freedom. Indeed, in this paper we will exhibit a Virasoro-Witt algebra corresponding to the variations of the conformal structure of $\mathcal{L}$ within the discretized moduli space mentioned above. The full symmetry algebra is the product of the Kac-Moody algebra associated with the Geroch group and this Virasoro-Witt algebra (this algebra has been identified previously for topologically trivial world-sheets [23], but there the extra symmetries do not generate new solutions beyond those generated by the Geroch group). In addition, the Geroch group itself may also change the topology of the world-sheet. This happens whenever the function $\mathcal{E}(\xi, \xi)$ has a singularity in the $\xi$-plane, where the conformal factor $f^{-1} \mathrm{e}^{2 k}$ has a branch point; the world-sheet is then a covering of $\mathcal{L}$ with these branch points.

This paper is organized as follows. In Section 2 we explain the construction of flat metrics on an arbitrary Riemann surface and the nature of their singularities. In Section 3 we apply these results to the construction of topologically non-trivial flat fourdimensional manifolds with cosmic string singularities. Periodic solutions of the Ernst equations are treated in Section 4, where we show how to generate periodic analogs of the known static axisymmetric solutions; we give only a sketchy account of the non-static case, however, as our results are still incomplete, requiring more sophisticated techniques. In Section 5, all these results are combined to derive the new solutions with non-trivial topology. Section 6 is devoted to a discussion of the new symmetries and the action of the Virasoro-Witt algebra on moduli space.

\section{Flat metrics on Riemann surfaces}

Hereafter we denote by $\mathcal{L}$ a Riemann surface of genus $g$ with local coordinates $(z, \bar{z})$. The metric on $\mathcal{L}$ may be chosen in many different ways. This is related to a well known and basic property of conformal field theories, including string theory, namely their invariance with respect to local variations of the metric. An especially convenient choice is based on the Mandelstam picture of string interactions in the light-cone gauge [3,4], where the metric is taken to be flat and the unitarity of the theory is manifest. Except for genus $g=1$, such a metric will unavoidably have $\delta$-function singularities of the curvature, yielding the standard Euler characteristic $\chi=2-2 g$ for a surface of genus $g$ 
(in the string theory interpretation, these singularities correspond to points where strings split or join). Note that the singularities can always be smoothed out by means of a conformal transformation of the metric. By contrast, the models considered here, which are obtained from a reduction of the Einstein equations in higher dimensions, are not conformally invariant ${ }^{4}$. Thus, the singular points cannot be eliminated here: this is the price we have to pay for topological non-triviality of the solutions.

As already explained in the introduction, we pick a meromorphic differential $\mathrm{d} \xi(P)$ and define a flat metric on $\mathcal{L}$ by (11). Away from the zeros and the poles of $\mathrm{d} \xi(P)$ and in accordance with (10), we can adopt $\xi(P)=x(P)+i \rho(P)$ as a local coordinate on any simply connected region of $\mathcal{L}$. Let us now discuss in turn the various situations arising from the different choices of $\mathrm{d} \xi(P)$. We remark that among these, the case where $\mathrm{d} \xi$ is a differential of the second kind (i.e. having higher order poles) is usually not considered in the string literature.

\section{1. $\mathrm{d} \xi$ is a differential of the first kind}

The linear space of holomorphic differentials (abelian differentials of the first kind) on $\mathcal{L}$ has complex dimension $g$. According to the Riemann-Roch theorem, any holomorphic differential $\mathrm{d} \xi$ on $\mathcal{L}$ possesses $2 g-2$ zeros at some points $P_{1}, \ldots, P_{2 g-2}$; for simplicity, we will assume that $\mathrm{d} \xi$ has only simple zeroes. Away from these points the curvature vanishes. In the neighbourhood of any zero $P_{j}$, the metric (11) is degenerate, and we have

$$
\mathrm{d} s_{0}^{2}=C|z|^{2} \mathrm{~d} z \mathrm{~d} \bar{z}, \quad C>0 \text { as } P \sim P_{j},
$$

where $z(P)$ is a local coordinate in the neighbourhood of $P_{j}$ such that $z\left(P_{j}\right)=0$. The Gaussian curvature of an arbitrary metric $q(z, \bar{z}) \mathrm{d} z \mathrm{~d} \bar{z}$ has the form

$$
\mathcal{K}=-\frac{(\log q)_{z \bar{z}}}{2 q}
$$

and the Euler characteristic of $\mathcal{L}$ is defined by

$$
\chi=\frac{1}{2 \pi} \int_{\mathcal{L}} q \mathcal{K} \mathrm{d} z \mathrm{~d} \bar{z} .
$$

Substituting the above form of the metric near $P_{j}$, we find

$$
q \mathcal{K}=-\frac{1}{2}\left(\log |z|^{2}\right)_{z \bar{z}}=-2 \pi \delta(u) \delta(v), \quad u \equiv \operatorname{Re} z, \quad v \equiv \operatorname{Im} z,
$$

and therefore the integral (13) receives a contribution $(-1)$ from each singular point; these contributions add up to the expected result $\chi=2-2 g$. Geometrically, the different flat pieces of $\mathcal{L}$ are glued together at the "interaction points" $P_{j}$ [4]. Singularities are

\footnotetext{
${ }^{4}$ At least not in the usual sense. However, we would like to draw the reader's attention to the fact that in many respects the dimensionally reduced theory resembles Liouville theory, where the conformal factor also does not decouple, but conformal invariance can be restored nonetheless at the quantum level. See also Ref. [24] for a discussion.
} 
absent only for the torus, for which $2-2 g=0$; there is then only one holomorphic differential $\mathrm{d} z$. To be able to interpret these "naked singularities" and the singularities of the associated four-manifold in Section 3, we introduce polar coordinates in the vicinity of $P_{j}$ by

$$
r=|z|, \quad \theta=\arg z .
$$

Defining a new radial coordinate $R=\frac{1}{2} \sqrt{C} r^{2}$, the metric (12) near $P_{j}$ is cast into the form

$$
\mathrm{d} s_{0}^{2}=\mathrm{d} R^{2}+4 R^{2} \mathrm{~d} \theta^{2} .
$$

\section{2. $\mathrm{d} \xi$ is a differential of the second kind}

Next consider a meromorphic differential $\mathrm{d} \xi$ on $\mathcal{L}$ with poles of order $>1$ only. For simplicity, we assume that $\mathrm{d} \xi$ has only poles of order two located at the points $R_{1}, \ldots$, $R_{n}$ :

$$
\mathrm{d} \xi(P)=C_{j} \frac{\mathrm{d} z}{z^{2}}+O(1), \quad \text { as } P \sim R_{j}, j=1, \ldots, n .
$$

Introducing a new complex coordinate $\zeta=-z^{-1}$ in the neighbourhood of $R_{j}$, we get

$$
\mathrm{d} s_{0}^{2}=\tilde{C} \mathrm{~d} \zeta \mathrm{d} \bar{\zeta} ; \quad \zeta \sim \infty \text { as } P \sim R_{j} .
$$

Consequently, a small disc around the point $R_{j}$ represents an asymptotically flat region on $\mathcal{L}$; the number of such regions is equal to $n$. The number of zeroes of $\mathrm{d} \xi$ on $\mathcal{L}$ is now equal to $2 g-2+2 n$; the behaviour of the metric at these points was discussed in the previous subsection. As a special case, we can have $\mathrm{d} \xi=\mathrm{d} h(P)$, where $h(P)$ is some meromorphic function on $\mathcal{L}$ (here with simple poles); then both functions $x=\operatorname{Re} h(P)$ and $\rho=\operatorname{Im} h(P)$ are single-valued on $\mathcal{L}$. We do not know whether differentials of the second kind admit an interpretation in the context of string theory.

\section{3. $\mathrm{d} \xi$ is a differential of the third kind}

Finally, consider abelian differentials of the third kind, having first order poles at the points $Q_{1}, \ldots, Q_{n}$ with purely imaginary residues (this is sufficient for our purposes):

$$
\mathrm{d} \xi(P)=\left(-\frac{i \alpha_{j}}{z}+O(1)\right) \mathrm{d} z, \quad \alpha_{j} \in \mathbb{R} \quad \text { as } P \sim Q_{j} ; \sum_{j} \alpha_{j}=0 .
$$

For $\xi(P)$ close to $Q_{j}$ we have

$$
\xi(z) \sim-i \alpha_{j} \log z \quad \text { as } z \sim 0,
$$

whence

$$
x \sim \alpha_{j}(\arg z+2 \pi k), \quad \rho \sim \alpha_{j} \log |z|,
$$


where $k \in \mathbb{Z}$. Thus the small disc centered at $Q_{j}$ represents a semi-infinite tube such that $\rho$ increases logarithmically to infinity as one moves along the tube and $x$ parametrizes the transverse direction (and is thus only defined modulo $2 \pi \alpha_{j}$ ).

Relation (15) defines $d \xi$ only up to an arbitrary linear combination of holomorphic differentials. However, if we impose the additional restriction that all cyclic periods of $\mathrm{d} \xi$ are real (so there are $2 g$ real conditions), it will be uniquely defined. In this case $\rho \equiv \operatorname{Im} \xi$ is a single-valued function on $\mathcal{L}$. This is precisely the differential used in the Mandelstam description of multiple string scattering in the light-cone gauge; the globally defined coordinate $\rho$ plays the role of light-cone time, and the multi-valued coordinate $x$ parametrizes the various strings at any given time. Since the number of zeroes of an arbitrary meromorphic differential is equal to the number of its poles plus $2 g-2, d \xi$ has altogether $2 g-2+n$ zeroes on $\mathcal{L}$, which correspond to the string interaction points as already explained. The semi-tubes growing out of the surface near $Q_{j}$ are interpreted as asymptotic in- or out-states of free strings, depending on whether the residue is positive or negative (the residues are identified with the momenta of the in- and outgoing strings, so the vanishing of their sum expresses nothing but momentum conservation).

When discussing solutions of the Ernst equation on $\mathcal{L}$, we can, of course, also allow for linear combinations of differentials of the the second and third kinds.

\section{Simple examples of topologically non-trivial flat manifolds}

Before discussing the Ernst equation on $\mathcal{L}$, we find it instructive to explain how our construction works for $\mathcal{E}=1$, leading to topologically non-trivial flat manifolds $\mathcal{M}$ in four dimensions, i.e. with metric

$$
\mathrm{d} s^{2}=\mathrm{d} \xi \mathrm{d} \bar{\xi}+\rho^{2} \mathrm{~d} \phi^{2}-\mathrm{d} t^{2},
$$

where the notation is the same as in the previous sections. Since all examples are topologically the product of the time axis $\mathbb{R}$ and a spatial flat three-manifold $\mathcal{M}_{0}$, we can effectively ignore the time coordinate in the sequel. Evidently, for the metric (16) to be well defined, it is necessary that $\rho(P)$ be globally defined. This shows again that all cyclic periods of $\mathrm{d} \xi(P)$ should be real. Owing to the fact that the period matrix associated with $\mathcal{L}$ has positive imaginary part, this condition cannot be satisfied for holomorphic $\mathrm{d} \xi(P)$. Consequently, $\mathrm{d} \xi(P)$ must have poles of some kind. Its singular parts and the requirement of reality determine $\mathrm{d} \xi$ uniquely. We now discuss the properties of $\mathcal{M}_{0}$ taking into account the possible choices of abelian differentials $\mathrm{d} \xi$.

Suppose that $\mathrm{d} \xi(P)$ has a zero at $Q \in \mathcal{L}$. In the vicinity of $Q$, the world-sheet metric can be brought into the form (14), so the associated metric on $\mathcal{M}_{0}$ becomes

$$
\mathrm{d} s^{2}=\mathrm{d} R^{2}+4 R^{2} \mathrm{~d} \theta^{2}+\rho^{2} \mathrm{~d} \phi^{2},
$$

where $\rho$ at $R=0$ is given by $\rho(Q)$, and $R$ and $\theta$ are local polar coordinates in the $\xi$-plane. If the second term were $R^{2} \mathrm{~d} \theta^{2}$, the space would obviously be flat. On the other hand, if instead of $\beta^{2}=4$, the prefactor obeyed $\beta^{2}<1$, the metric would correspond to a cosmic string with deficit angle $2 \pi(1-\beta)$ around $R=0$ [25]. In the case at hand, 
there is an excess angle of $2 \pi$ instead. Accordingly, we identify this singularity with a cosmic string of negative tension at $R=0$; since $0 \leqslant \phi<2 \pi$, this cosmic string forms a ring of radius $\rho(Q)$. By construction, there are as many cosmic strings as there are zeroes of $\mathrm{d} \xi$ in the domain of positive $\rho$. Encircling such a string once, one ends up in another (flat) axisymmetric "universe", while a rotation of $4 \pi$ will bring the observer back to the universe from where he or she started; this is the physical meaning of the excess angle. Analogously, the number of different "universes" that can be reached in this fashion increases with the order of the zero (it is easy to see that the excess angle is then $4 \pi, 6 \pi, \ldots)$, and for each extra zero of $d \xi$, new separate "universes" become accessible.

The properties of the metric (16) near the poles of $\mathrm{d} \xi$ can be likewise understood on the basis of the results of Section 2. For instance, let $P_{0}$ be a second order pole of $\mathrm{d} \xi$, and $z=z(P)$ a local coordinate at $P_{0}$ defined in such a way that $\mathrm{d} \xi \sim z^{-2} \mathrm{~d} z$ as $P \sim P_{0}$. Clearly, $\xi=z^{-1} \rightarrow \infty$ as $P \rightarrow P_{0}$, implying $\rho(z)=\operatorname{Im}\left[z^{-1}\right]$ and $x(z)=\operatorname{Re}\left[z^{-1}\right]$. The small disc around the second order pole of $\mathrm{d} \xi$ corresponds to an asymptoticaly flat region on $\mathcal{M}_{0}$ (the same conclusion holds for poles of yet higher order). On the other hand, if $P_{0}$ is a simple pole of $\mathrm{d} \xi$ with imaginary residue, a small disc on $\mathcal{L}$ centered at $P_{0}$ is topologically equivalent to a semi-infinite tube. The domain in $M_{0}$ corresponding to this tube is the product of the domain $\rho>\rho_{0}$ in the $(\rho, \phi)$-plane and a circle $S^{1}$ in terms of the $x$-coordinate ( $\rho_{0}$ is a constant related to diameter of the disc). In other words, the global structure of $\mathcal{M}_{0}$ can be described as follows. If $\mathcal{L}$ were just the complex plane with coordinates $(x, \rho)$ we would get the space $\mathcal{M}_{0}=\mathbb{R}^{3}$ by rotating the half-plane $\rho \geqslant 0$ around the symmetry axis $\rho=0$. This procedure may be repeated for arbitrary $\mathcal{L}$. Consider the part $\mathcal{L}^{+}$of $\mathcal{L}$ for which $\rho \geqslant 0$; then $\mathcal{M}_{0}$ is obtained by "rotating" $\mathcal{L}^{+}$ around the contour $\mathcal{C}$ consisting of all points $P \in \mathcal{L}$ such that $\rho(P)=0$ (observe that $\mathcal{C}$ may have several disconnected components). Rigorously speaking, we get a foliation $\mathcal{M}_{0} \rightarrow \mathcal{L}^{+}$where the fibre corresponding to each point of $\mathcal{L}^{+}$is a circle of radius $\rho$. The flat metric is properly defined on all of $\mathcal{M}_{0}$ and regular away from the poles and zeroes of $\mathrm{d} \xi$ as we already explained. The total number of cosmic strings, asymptotic regions and semi-infinite tubes is constrained by the Riemann-Roch theorem according to Section 2.

Notice that in our construction the subset $\mathcal{L}_{-} \subset \mathcal{L}$ corresponding to $\rho<0$ can be ignored, because we admit only non-negative $\rho$ in order to be able to interpret the metric as a genuine metric in four dimensions. Taking into account the reflection symmetry $\rho \rightarrow-\rho$ on $\mathcal{M}$ of the metric (16), the section $\phi=k \pi, k=1,2$ of $\mathcal{M}_{0}$ may be identified with $\mathcal{L}^{+}$glued to its mirror image along the "symmetry axis" $\rho=0$. So without loss of generality we could have assumed from the beginning that the curve $\mathcal{L}$ admits an anti-holomorphic involution $\tau: \mathcal{L} \rightarrow \mathcal{L}, \tau^{2}=1$ such that $\tau\left(\mathcal{L}^{+}\right)=\mathcal{L}^{-}$and could have defined $\mathcal{C}$ as the set of points invariant with respect to $\tau$ (locally, the involution $\tau$ maps $(x, \rho)$ to $(x,-\rho))$. In this case the section $\phi=k \pi, k=0,1$ of $\mathcal{M}_{0}$ may be identified with the surface $\mathcal{L}$ itself.

The above considerations can be extended in an obvious manner to the simpler case of three-manifolds with metrics

$$
\mathrm{d} s^{2}=\mathrm{d} \xi \mathrm{d} \bar{\xi}+\mathrm{d} x_{3}^{2}
$$


whose third coordinate $x_{3}$ is unrelated to $\xi(P)$; from the two-dimensional point of view, we are dealing here with a constant dilaton. The essential difference is that we now need not require real periods. Topologically, $\mathcal{M}_{0}$ is then a product $\mathcal{L} \times \mathbb{R}$ or $\mathcal{L} \times S^{\mathrm{I}}$.

In a somewhat different context, flat Lorentzian three-manifolds having non-singular space-like surfaces at any finite value of the time parameter, but initial and final singularities at $t=0$ and $t=\infty$, were constructed in Ref. [26].

\section{Periodic solutions of the Ernst equation}

Since our construction requires periodic solutions of the Ernst equation, we will in this section explicitly demonstrate that a large class of solutions actually exists (the periodic analog of the Schwarzschild solution was already constructed in Ref. [27]). While so far we have explicit examples only for the static case (i.e. $A=0$ in (8)), we give arguments why periodic solutions with rotation, and in particular a periodic analog of the Kerr solution, should also exist; however, their explicit construction requires more sophisticated tools from the theory of integrable systems.

\subsection{Static case}

As is well known, static metrics with $A=0$ correspond to real Ernst potentials. For these, the Ernst Eq. (5) can be linearized by the substitution

$$
\omega=\log \mathcal{E},
$$

and is thereby reduced to the Euler-Darboux equation

$$
\omega_{x x}+\frac{1}{\rho} \omega_{\rho}+\omega_{\rho \rho}=0 .
$$

The metric (6) becomes

$$
\mathrm{d} s^{2}=\mathrm{e}^{-\omega}\left[\mathrm{e}^{2 k}\left(\mathrm{~d} x^{2}+\mathrm{d} \rho^{2}\right)+\rho^{2} \mathrm{~d} \phi^{2}\right]+\mathrm{e}^{\omega} \mathrm{d} t^{2},
$$

where the conformal factor is determined from the first order equation

$$
k_{\xi}=\frac{i \rho}{2}\left(\omega_{\xi}\right)^{2}
$$

or, equivalently,

$$
k_{\rho}=\frac{\rho}{4}\left(\omega_{\rho}^{2}-\omega_{x}^{2}\right), \quad k_{x}=\frac{\rho}{2} \omega_{x} \omega_{\rho}
$$

We can now construct $x$-periodic analogs of known static solutions by means of the following procedure.

Let $\omega_{0}(x, \rho)$ be some solution of (19). Consider the expression ${ }^{5}$

\footnotetext{
${ }^{5}$ The following construction is inspired by the construction of the Weierstrass $\wp$-function.
} 


$$
\omega(x, \rho)=\sum_{n=-\infty}^{\infty}\left\{\omega_{0}(x+n L, \rho)+a_{n}\right\},
$$

where the coefficients $a_{n}$ are constants to be chosen in such a way that series (22) becomes convergent. It is important that these coefficients do not depend on $(x, \rho)$ since otherwise the sum could not possibly satisfy the original equation (19). If convergence may be achieved, the function (22) describes a solution of (19) with period $L$. Clearly, in order to obtain a truly periodic metric (20) we must also verify the periodicity of the function $k(x, \rho)$ defined by $(21)$.

The following theorem shows that our method of construction is quite general.

Theorem 1. Let $\omega_{0}(x, \rho)$ be any solution of the Euler-Darboux equation corresponding to an asymptotically flat metric (20), i.e.

$$
\omega_{0}(x, \rho)=\frac{\beta}{r}+O\left(r^{-2}\right) \quad \text { as } r \rightarrow \infty,
$$

where $r=\sqrt{x^{2}+\rho^{2}} ; M=-\frac{1}{2} \beta$ is the mass. Let

$$
a_{n}=-\frac{\beta}{L|n|}, \quad n \neq 0, a_{0}=0 .
$$

Then series $(22)$ is convergent for all $(x, \rho)$ except the points $\left(x_{0}+n L, \rho_{0}\right)$, where the function $\omega_{0}(x, \rho)$ is singular $(n \in \mathbb{Z})$, and defines a periodic function with period $L$.

Proof. For large $n$ we have

$$
\omega_{0}(x+n L, \rho)+a_{n}=\beta\left(\frac{1}{\sqrt{(x+n L)^{2}+\rho^{2}}}-\frac{1}{L|n|}\right)+O\left(\frac{1}{n^{2}}\right)=O\left(\frac{1}{n^{2}}\right)
$$

by (23), and therefore the series (22) converges if $(x+n L, \rho)$ does not coincide with a singular point of $\omega_{0}(x, \rho)$ for any $n$.

So starting from an arbitrary static asymptotically flat solution we can construct its $x$ periodic analog. Consider for instance the Schwarzschild solution, which is characterized by the Ernst potential

$$
\omega_{0}=\log \mathcal{E}_{0}, \quad \mathcal{E}_{0}(x, \rho)=\frac{\sqrt{(x-M)^{2}+\rho^{2}}+\sqrt{(x+M)^{2}+\rho^{2}}-2 M}{\sqrt{(x-M)^{2}+\rho^{2}}+\sqrt{(x+M)^{2}+\rho^{2}}+2 M},
$$

where $M \in \mathbb{R}$ is an arbitrary positive constant (the mass of the black hole). Here, all square roots are taken to be positive; this means that we do not consider (25) inside the event horizon, which coincides with the segment $\rho=0, x \in[-M, M]$. The coefficient $\beta$ in (23) is therefore equal to $-2 M$. Thus the periodic analog of the Schwarzschild solution (25) has the following form:

$$
\mathcal{E}(x, \rho)=\mathcal{E}_{0}(x, \rho) \prod_{n=1}^{\infty} \mathcal{E}_{0}(x+n L, \rho) \mathcal{E}_{0}(x-n L, \rho) \exp \left(\frac{4 M}{n L}\right) .
$$




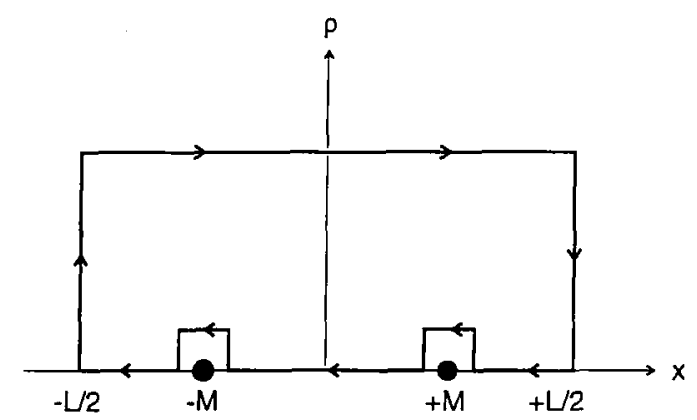

Fig. 1. Integration contour used in the proof of (27).

Obviously,

$$
\mathcal{E}(x+L, \rho)=\mathcal{E}(x, \rho) .
$$

This is essentially the solution found in Ref. [27]. In the sequel we will assume $\frac{1}{2} L>M$ (for $L \leqslant 2 M$, the interpretation of the solution is not clear as the horizon overlaps with itself, and the Ernst potential vanishes on the symmetry axis). Convergence of the infinite product (26) is equivalent to convergence of the series (22) and thus guaranteed by Theorem 1 (for non-negative values of the square roots in (25)). Consequently, the solution (26) is a periodic function on the upper half plane $\rho \geqslant 0$ with "fundamental region" $\mathcal{F}$ defined by $\rho \geqslant 0,-\frac{1}{2} L \leqslant x \leqslant \frac{1}{2} L$.

It is now not difficult to verify that the function $\mathcal{E}(x, \rho)$ defined by $(26)$ is smooth everywhere on $\mathcal{F}$ away from the points $x= \pm M, \rho=0$. and non-zero everywhere except on the horizon (i.e. $\rho=0,|x| \leqslant M$ ). The periodicity of the conformal factor, i.e.

$$
k(x+L, \rho)=k(x, \rho)
$$

is implied by

$$
\int_{-L / 2}^{L / 2} k_{x} \mathrm{~d} x=0
$$

where the derivative $k_{x}$ is to be evaluated by means of (9). This, in turn is a consequence of the vanishing of the following contour integral:

$$
\int_{l}\left\{\frac{\rho}{4}\left(\omega_{\rho}^{2}-\omega_{x}^{2}\right) \mathrm{d} \rho+\frac{\rho}{2} \omega_{x} \omega_{\rho} \mathrm{d} x\right\},
$$

where the closed contour $l$ is depicted in Fig. 1. This integral vanishes because the function $\omega$ obeys (19) and is smooth everywhere inside of $l$. The integrals along the edges $\left[\left(-\frac{1}{2} L, 0\right),\left(-\frac{1}{2} L, \rho\right)\right]$ and $\left[\left(\frac{1}{2} L, \rho\right),\left(\frac{1}{2} L, 0\right)\right]$ cancel due to the periodicity of $\omega(x, \rho)$. Owing to the presence of the factor $\rho$ in $(28)$ the contribution of the interval 
$\left[\left(\frac{1}{2} L, 0\right),\left(-\frac{1}{2} L, 0\right)\right]$ reduces to a sum of contributions of two small rectangular paths around the points $x=-M$ and $M$ (cf. Fig. 1), where the derivatives $\omega_{x}$ and $\omega_{\rho}$ become singular. These contributions cancel by virtue of the symmetry

$$
\omega(-x, \rho)=\omega(x, \rho)
$$

inherited by solution $\mathcal{E}$ from $\mathcal{E}_{0}$. So the integral along the contour $\left[\left(-\frac{1}{2} L, \rho\right),\left(\frac{1}{2} L, \rho\right)\right]$ also vanishes and we get $k\left(-\frac{1}{2} L, \rho\right)=k\left(\frac{1}{2} L, \rho\right)$. In conclusion, the metric (20) corresponding to the periodic Ernst potential (26) is also periodic.

The asymptotic behavior of the Ernst potential (26) is given by

$$
\mathcal{E}=C \rho^{4 M / L}(1+o(1)) \text { as } \rho \rightarrow \infty,
$$

where $C$ is some constant. To see this, recall that the function $\omega=\log \mathcal{E}$ is defined by

$$
\omega(x, \rho)=\omega_{0}(x, \rho)+\sum_{n=1}^{\infty}\left[\omega_{0}(x+n L, \rho)+\omega_{0}(x-n L, \rho)+\frac{4 M}{n L}\right] .
$$

Substituting the explicit expression for $\omega_{0}(x, \rho)(25)$ and differentiating with respect to $\rho^{2}$, we obtain

$$
\frac{\partial \omega}{\partial\left(\rho^{2}\right)}(x, \rho)=\sum_{n=-\infty}^{\infty} \frac{2 M\left[s_{1}(n)+s_{2}(n)\right]}{\left[s_{1}(n)+s_{2}(n)+2 M\right]\left[s_{1}(n)+s_{2}(n)-2 M\right] s_{1}(n) s_{2}(n)},
$$

where $s_{1}(n)=\sqrt{(x+n L+M)^{2}+\rho^{2}}$ and $s_{2}(n)=\sqrt{(x+n L-M)^{2}+\rho^{2}}$. The leading term in this series for large $\rho$ can be estimated by approximating the sum by an integral; it is given by

$$
\sum_{n=-\infty}^{\infty} \frac{M}{\left((x+n L)^{2}+\rho^{2}\right)^{3 / 2}}=\frac{2 M}{L} \frac{1}{\rho^{2}}(1+o(1)) .
$$

Thus,

$$
\omega=\frac{2 M}{L} \log \rho^{2}+O(1) \quad \text { as } \rho \rightarrow \infty
$$

and $\mathcal{E}=C \rho^{4 M / L}(1+o(1))$ for some constant $C$. Hence, as $\rho \rightarrow \infty$, the metric (20) tends to the Kasner solution

$$
\mathrm{d} s^{2}=\tilde{C} \rho^{\alpha^{2} / 2-\alpha}\left(\mathrm{d} x^{2}+\mathrm{d} \rho^{2}\right)+C^{-1} \rho^{2-\alpha} \mathrm{d} \phi^{2}-C \rho^{\alpha} \mathrm{d} t^{2},
$$

where $\tilde{C}$ is another constant of integration and the Kasner parameter $\alpha$ is related to the period $L$ by $\alpha=4 M L^{-1}$, so that $0 \leqslant \alpha<2$ with our assumption on the range of $M$.

The solution (26) has a compact event horizon coinciding with the segment $\rho=$ $0,-M \leqslant x \leqslant M$. Outside the horizon it is everywhere non-singular, including the segment of the symmetry axis outside the horizon. Using the standard product representation for the $\Gamma$-function, we find

$$
\mathcal{E}(x, \rho=0)=\exp \left(\frac{4 \gamma M}{L}\right) \frac{\Gamma((|x|+M) / L) \Gamma(1-(|x|-M) / L)}{\Gamma((|x|-M) / L) \Gamma(1-(|x|+M) / L)}
$$


for $M \leqslant|x| \leqslant \frac{1}{2} L$ ( $\gamma$ is the Euler constant), and $\mathcal{E} \equiv 0$ for $\rho=0$ and $|x| \leqslant M$. As a consequence of the reflection and translation symmetry, the free integration constant in Eq. (14) may be chosen in such a manner that conical singularities on the part of the symmetry axis outside the horizon are avoided (this requirement fixes the constant $\tilde{C}$ in (30)). In the limit $L \rightarrow \infty$, the solution obviously tends (pointwise) to the ordinary Schwarzschild solution. The leading term in the asymptotic expansion then approaches the flat metric, as it should be ${ }^{6}$.

By theorem 1 we can obtain a periodic counterpart of any asymptotically flat static solution of (5). The method described in this section fails, however, for non-static axisymmetric stationary solutions, because the Ernst equation can no longer be linearized in this case. Since the extension requires entirely new techniques, we shall sketch a possible method based on methods borrowed from soliton theory in the next section.

\subsection{General case}

The Ernst equation (5) may be derived as the compatibility condition of the following linear system [6,7,10] (for more recent developments, see Refs. [20,16], and Ref. [11], whose notation and conventions we will follow in this section):

$$
\begin{aligned}
\Psi_{\xi} & =U \Psi, \quad \Psi_{\bar{\xi}}=V \Psi, \\
U & =\left(\begin{array}{ll}
C_{1} & 0 \\
0 & C_{2}
\end{array}\right)+\sqrt{\frac{\lambda-\bar{\xi}}{\lambda-\xi}}\left(\begin{array}{lr}
0 & C_{1} \\
C_{2} & 0
\end{array}\right), \\
V & =\left(\begin{array}{cc}
C_{3} & 0 \\
0 & C_{4}
\end{array}\right)+\sqrt{\frac{\lambda-\xi}{\lambda-\bar{\xi}}}\left(\begin{array}{lr}
0 & C_{3} \\
C_{4} & 0
\end{array}\right),
\end{aligned}
$$

where $\lambda \in \mathbb{C}$ is the spectral parameter ${ }^{7} ; \Psi(\lambda, \xi, \bar{\xi})$ is a two-by-two matrix, whose coefficients $C_{j}(\xi, \bar{\xi})$ are related to the Enst potential $\mathcal{E}$ by

$$
C_{1}=\frac{\mathcal{E}_{\xi}}{\mathcal{E}+\overline{\mathcal{E}}}, \quad C_{2}=\frac{\overline{\mathcal{E}}_{\xi}}{\mathcal{E}+\overline{\mathcal{E}}}, \quad C_{3}=\frac{\mathcal{E}_{\bar{\xi}}}{\mathcal{E}+\overline{\mathcal{E}}}, \quad C_{4}=\frac{\overline{\mathcal{E}}_{\bar{\xi}}}{\mathcal{E}+\overline{\mathcal{E}}} .
$$

The identification (33) is a consequence of the asymptotic condition

$$
\Psi(\lambda=\infty)=\left(\begin{array}{cc}
1 & \mathcal{E} \\
-1 & \overline{\mathcal{E}}
\end{array}\right)
$$

\footnotetext{
${ }^{6}$ Altematively, one could regard this solution as describing an infinite chain of black holes spaced at a distance $L$. At first sight, it seems remarkable that this configuration does not require conical singularities on the axis between adjacent black holes for stability. Rather, it appears to be stabilized by its symmetry under reflections and translations and the presence of infinitely many black holes "on each side". However, this also indicates instability under non-periodic perturbations, which makes this interpretation somewhat less attractive.

${ }^{7}$ The spectral parameter $\lambda$ is called $w$ in Refs. $[20,16]$. Sometimes it is convenient to use the $(x, \rho)$ dependent spectral parameter $t=(1 / \rho)\left(\lambda-x+\sqrt{(\lambda-x)^{2}+\rho^{2}}\right.$ (first introduced in Ref. [6]) mapping $\mathcal{S}$ to the complex plane.
} 
Considered as a function of the complex spectral parameter $\lambda$ with fixed $(\xi, \bar{\xi})$, the linear system (32) lives on the two-sheeted Riemann surface $\mathcal{S}$ of genus zero defined by the equation

$$
\gamma^{2}=(\lambda-\xi)(\lambda-\bar{\xi}) ;
$$

$\Psi$ is thus a function of the three variables $(P, x, \rho)$, where $P \in \mathcal{S}$ (actually, we should distinguish points on the spectral curve $\mathcal{S}$ from points on the world-sheet $\mathcal{L}$, but we will nevertheless use letters $P, Q, \ldots$ if there is no danger of confusion). By $\sigma$, we denote the involution on $\mathcal{S}$ interchanging the two sheets.

The existence of the linear system (32) permits us to construct solutions of (1) by means of the inverse scattering method [6-14,17,20]. So far, most efforts in this direction have been concerned with the investigation of asymptotically flat solutions of (5). Although our results are still incomplete, we now present some evidence that every asymptotically flat solution of (5) should have a natural periodic counterpart.

To illustrate these arguments, let us attempt to construct the periodic analog of the Kerr solution. For this purpose, recall the standard construction of $2 n$-soliton solutions of (5) (there are no solutions with an odd number of solitons). The associated function $\Psi_{n}(P)$ has the following form:

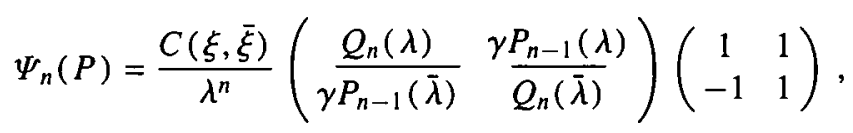

where

$$
Q_{n}(\lambda)=\sum_{k=1}^{n} q_{k} \lambda^{k}, \quad P_{n-1}(\lambda)=\lambda^{k-1}+\sum_{k=1}^{n-2} p_{k} \lambda^{k}
$$

are two polynomials with $(x, \rho)$-dependent coefficients $p_{k}, q_{k} ; C(\xi, \bar{\xi})$ is a diagonal matrix providing normalization of the first column according to (34): $C_{11}=\left(q_{n}-1\right)^{-1}$; $C_{22}=\left(1-\bar{q}_{n}\right)^{-1}$. For arbitrary coefficient functions $p_{k}$ and $q_{k}$, the function $\Psi_{n}$ will not satisfy the linear system (32) because its logarithmic derivatives $\Psi_{\xi} \Psi^{-1}$ and $\Psi_{\bar{\xi}} \Psi^{-1}$ will have not only the poles at $\lambda=\xi$ and $\lambda=\bar{\xi}$ required by (32), but also at the zeroes $\lambda_{1}, \ldots, \lambda_{2 n}$ of det $\Psi_{n}$. To eliminate these additional poles suppose that the points $\lambda_{1}$, $\ldots, \lambda_{2 n}$ are independent of $(\xi, \bar{\xi})$, and that the eigenvectors of $\Psi_{n}$ at these points are also $(\xi, \bar{\xi})$-independent, i.e.

$$
\Psi_{n}\left(\lambda_{k}\right)\left(\begin{array}{c}
1 \\
d_{k}
\end{array}\right)=0, \quad k=1, \ldots, 2 n,
$$

where $d_{k}$ are some constants. From (36) we can deduce $4 n$ linear equations for the $2 n$ functions $p_{0}, \ldots, p_{n-2}, q_{0}, \ldots, q_{n}$. To ensure their solvability it is enough to require that

$$
\lambda_{2 k-1}=\bar{\lambda}_{2 k-1}, \quad \lambda_{2 k}=\bar{\lambda}_{2 k}, \quad d_{2 k-1}=-\bar{d}_{2 k-1}, \quad d_{2 k}=-\bar{d}_{2 k}
$$

or

$$
\lambda_{2 k-1}=\bar{\lambda}_{2 k}, \quad d_{2 k-1}=-\bar{d}_{2 k}
$$


for $k=1, \ldots, n$.

The related Ernst potential is then easily identified as

$$
\mathcal{E}=\frac{q_{n}+1}{q_{n}-1},
$$

where $q_{n}$, by Kramer's rule, is the ratio of two $2 n \times 2 n$ determinants. This expression is more conveniently dealt with in terms of the potential

$$
\Gamma \equiv \frac{1-\mathcal{E}}{1+\mathcal{E}}=-\frac{1}{q_{n}} .
$$

We will not write down the corresponding expressions for arbitrary $n$; they can be found in Ref. [28].

For $n=1$ and $\lambda_{1,2} \in \mathbb{R}, d_{1,2} \in i \mathbb{R}$ we get

$$
\Gamma^{-1}=\frac{c_{1}-c_{2}}{2} X+\frac{c_{1}+c_{2}}{2} Y,
$$

where

$$
c_{j} \equiv \frac{-1+d_{j}}{1+d_{j}}, \quad j=1,2,
$$

are new arbitrary constants satisfying $\left|c_{j}\right|=1$, and

$$
\begin{aligned}
& X \equiv \frac{1}{\lambda_{1}-\lambda_{2}}\left[\sqrt{\left(\lambda_{1}-\xi\right)\left(\lambda_{1}-\bar{\xi}\right)}+\sqrt{\left(\lambda_{2}-\xi\right)\left(\lambda_{1}-\bar{\xi}\right)}\right], \\
& Y \equiv \frac{1}{\lambda_{1}-\lambda_{2}}\left[\sqrt{\left(\lambda_{1}-\xi\right)\left(\lambda_{1}-\bar{\xi}\right)}-\sqrt{\left(\lambda_{2}-\xi\right)\left(\lambda_{1}-\bar{\xi}\right)}\right]
\end{aligned}
$$

are prolate ellipsoidal coordinates. This is nothing but the well known Kerr-NUT solution; to get the Kerr solution itself, we only have to put $c_{2}=-\overline{c_{1}} \equiv c$. To recover the multi-Kerr solution with arbitrary parameters, we have to choose $c_{2 j-1}=-\bar{c}_{2 j}$, $\left|c_{2 j-1}\right|=\left|c_{2 j}\right|=1$ for all $j=1, \ldots, g$.

Now let us turn to possible periodic generalizations. Intuitively, one would expect a periodic analog of the Kerr solution to represent an infinite superposition of identical Kerr black holes lined up on the symmetry axis at equal distances. Analytically, for an arbitrary solution of (5) obeying the periodicity condition (46), the associated linear system matrices $U$ and $V$ from (32) must satisfy the relations

$$
U(\lambda+L, x+L, \rho)=U(\lambda, x, \rho), \quad V(\lambda+L, x+L, \rho)=V(\lambda, x, \rho) .
$$

Then for a solution $\Psi$ of (32) we have

$$
\Psi(\lambda+L, x+L, \rho)=\Psi(\lambda, x, \rho) R(\lambda),
$$

where $R(\lambda)$ is some $(x, \rho)$-independent matrix. We emphasize that the world-sheet coordinate $x$ and the spectral parameter $\lambda$ must be shifted simultaneously by the same amount in order to maintain the $\lambda$-dependence prescribed by the linear system (32). 
Note also that, for topologically non-trivial world-sheets $\mathcal{L}, \Psi$ is not single-valued on $\mathcal{L}$ in general even for $R(\lambda)=1$.

From (40) we infer that the $(x, \rho)$-independent parameters in $\Psi$ must be invariant under the transformation $\lambda \rightarrow \lambda+L$ (in the general multi-soliton case, these parameters include the positions of the points $\lambda_{j}$ and the constants $c_{j}$ ). To construct a periodic analog of the Kerr solution we thus have to start from an infinite-soliton solution where the zeroes of det $\Psi$ are located at the points $\lambda_{\mathrm{I}}+n L$ (with the same parameter $c_{1}$ ) and $\lambda_{2}+n L$ (with the same paramter $c_{2}=-\overline{c_{1}}$ ). Just as for the periodic Schwarzschild solution, we can avoid "horizon overlap" by imposing the condition $L>2 M$, where $2 M \equiv\left|\lambda_{1}-\lambda_{2}\right|$.

A rigorous way to construct such a solution would be to consider a sequence of Ernst potentials $\mathcal{E}_{n}(x, \rho), n=1,2, \ldots$, where $\mathcal{E}_{n}$ is the $4 n+2$-soliton solution of (5) corresponding to the zeroes of det $\Psi$ at $\lambda_{1}+n L$ with the "dressing parameters" $c(|c|=1)$ and $\lambda_{2}+n L$ with the "dressing parameters" $-\bar{c}$. If we could prove the pointwise convergence of the sequence $\mathcal{E}_{n}$, the limit solution $\mathcal{E}=\lim _{n \rightarrow \infty} \mathcal{E}_{n}$ could be regarded as a periodic analog of Kerr solution. We have at this time no rigorous proof of convergence, but can offer the following arguments in favor of our hypothesis. First of all, the sequence is certainly convergent when $c_{2}=-c_{1}=1$, in which case the "dressing procedure" would reproduce the periodic analog of the Schwarzschild solution derived in the foregoing section. Secondly, for large $\rho$, the solution should describe the gravitational field of an infinitely extended rotating cylinder on the symmetry axis, a solution which is known to exist [28] (the periodic Schwarzschild solution likewise approaches the field of an infinite static cylinder). Moreover, the contribution from angular momentum to the asymptotic form of the Ernst potential at $\rho \rightarrow \infty$ is down by one order in comparison with the contribution of mass (i.e. of order $O\left(\rho^{-2}\right)$ rather than $O\left(\rho^{-1}\right)$ ), so they would not affect the convergence of the series. Indeed, these arguments suggest that the solution with rotation again approaches the Kasner solution (30) with the same Kasner parameter. However, even assuming convergence of the sequence $\mathcal{E}_{n}$, it is not clear at the moment whether the "dressing procedure" will not generate unphysical singularities outside the event horizon $\left[\lambda_{1}+n L, \lambda_{2}+n L\right]$ unlike in the static case, where such additional singularities could be shown to be absent.

Analogously, in order to construct periodic analogs of the algebro-geometrical solutions of (5), which contain the multi-soliton solutions as a degenerate partial case $[11,14]$ ), one would have to start from a hyperelliptic algebraic curve of infinite genus with a periodic configuration of immovable branch cuts.

The general conjecture is that every asymptotically flat solution of the Ernst equation with mass $M$ has a natural periodic analog which is asymptotically Kasner with Kasner parameter $4 M / L$. The spectral data of the fully periodic solution are obtained by shifting the spectral data of the initial solution by $n L$ for $n \in \mathbb{Z}$. In the limit $L \rightarrow \infty$ the periodic solution tends (pointwise) to its asymptotically flat counterpart. Assuming our conjecture to be true, the same correspondence should hold between the symmetry groups pertaining to asymptotically flat and periodic solutions, respectively, i.e. there should exist a "periodic Geroch group", also possessing a central extension. The corresponding Lie algebras should, of course, be the same (i.e. coincide with the affine Kac-Moody algebra $A_{1}^{(\mathrm{l})}$ ). 
Note that the periodicity constraint (40) for $\Psi$ implies the periodicity of all related quantities depending either on only $\lambda$ or on only $(\xi, \bar{\xi})$ (we can arrange $R=I$ by a suitable transformation $\Psi \rightarrow \Psi C(\lambda)$ ). For instance, with $F(\lambda)=I$ in (34), we get $\Psi(\lambda=\infty, \xi+L)=\Psi(\lambda=\infty, \xi)$, which implies periodicity of Ernst potential, i.e. $\mathcal{E}(x+L, \rho)=\mathcal{E}(x, \rho)$. Besides, it is easy to check that

$$
M(\lambda)=\frac{1}{\mathcal{E}+\overline{\mathcal{E}}} \Psi^{\prime}\left(\lambda^{\sigma}\right) \sigma_{1} \Psi(\lambda)
$$

(called "monodromy matrix" in Ref. [20]) is always $(\xi, \bar{\xi})$-independent. Hence, $M(\lambda)$ is periodic with period $L$ for $\Psi$ subject to (40) with $R(\lambda)=I$.

As an example, we calculate the monodromy matrix for the periodic Schwarzschild solution. The monodromy matrix associated with the ordinary Schwarzschild solution (25) has the form

$$
T_{0}=-\frac{(\lambda-M)(\lambda+M)}{\lambda^{2}} \sigma_{3}
$$

where we put $\lambda_{1}=-M, \lambda_{2}=M$ and $\sigma_{3}$ is the usual Pauli matrix. Now, for any static solution (i.e. $\mathcal{E}=\overline{\mathcal{E}}$ ), the related solution of the linear system (32) may be represented as

$$
\Psi(P)=\left(\begin{array}{cc}
\psi(P) & \psi\left(P^{\sigma}\right) \\
-\psi(P) & \psi\left(P^{\sigma}\right)
\end{array}\right),
$$

where $\psi(P)$ is a solution of

$$
\begin{aligned}
& (\log \psi)_{\xi}=\frac{1}{2}(\log \mathcal{E})_{\xi}\left(1+\sqrt{\frac{\lambda-\bar{\xi}}{\lambda-\xi}}\right), \\
& (\log \psi)_{\bar{\xi}}=\frac{1}{2}(\log \mathcal{E})_{\bar{\xi}}\left(1+\sqrt{\frac{\lambda-\xi}{\lambda-\bar{\xi}}}\right) .
\end{aligned}
$$

The related monodromy matrix is

$$
T=-\frac{\psi(P) \psi\left(P^{\sigma}\right)}{2 \mathcal{E}} \sigma_{3}
$$

If $\mathcal{E}_{1}$ and $\mathcal{E}_{2}$ are two different solutions of (5) with associated functions $\psi_{1}(P)$ and $\psi_{2}(P)$, and monodromy matrices $T_{1}(\lambda)$ and $T_{2}(\lambda)$, respectively, then $\mathcal{E}_{1} \mathcal{E}_{2}$ is a solution of (5) corresponding to $\psi(P)=\psi_{1}(P) \psi_{2}(P)$ with monodromy matrix $T(\lambda)=T_{1}(\lambda) T_{2}(\lambda)$. From this simple observation, we can derive the monodromy matrix corresponding to the solution (26) as an infinite product $\prod_{n=-\infty}^{\infty} T_{0}(\lambda+n L)$. Using the explicit form (41) of the matrix $T_{0}$, we obtain

$$
T(\lambda)=-\frac{\sin [\pi(\lambda-M) / L] \sin [\pi(\lambda+M) / L]}{\sin ^{2}[\pi \lambda / L]} \sigma_{3} .
$$

In the different parametrization adopted in Ref. [20], we would obtain $T(\lambda)=$ $\operatorname{diag}\left(r(\lambda), r^{-1}(\lambda)\right)$ instead, where $r(\lambda)=\sin [\pi(\lambda-M) / L] \sin ^{-1}[\pi(\lambda+M) / L]$. 
We observe the following difference between periodic and asymptotically flat solutions: in the asymptotically flat case, the dependence of the monodromy matrix on $\lambda$ can be inferred from the knowledge of the Ernst potential on the symmetry axis [20]. Comparison of the monodromy matrix for the periodic Schwarzschild solution with the related Ernst potential on the symmetry axis (cf. (31)) shows that this is no longer true for periodic solutions.

\section{The Ernst equation on $\mathcal{L}$}

We will now combine the results arrived at in the foregoing sections. That is, we will replace the flat metric (16) by the full metric (6), and at the same time assume the underlying world-sheet to be a non-trivial Riemann surface, thereby introducing a non-trivial gravitational field on the manifold $\mathcal{M}$. The non-flat metric on the background provided by (16) thus has the usual form of the metric on a stationary axisymmetric Einstein manifold, viz.

$$
\mathrm{d} s^{2}=f^{-1} \mathrm{e}^{2 k} \mathrm{~d} \xi \mathrm{d} \bar{\xi}+f^{-1}(\operatorname{Im} \xi)^{2} \mathrm{~d} \phi^{2}-f(\mathrm{~d} t+A \mathrm{~d} \phi)^{2},
$$

where the functions $f, k$ and $A$ depending only on $(\xi, \bar{\xi})$ may be expressed in terms of the complex-valued Ernst potential according to (9). The Einstein equations for the metric (42) reduce to the Ernst equation (5), together with (2) and (9), as already mentioned in the introduction.

Let us denote the poles of $\mathrm{d} \xi$ on $\mathcal{L}$ with non-zero residues by $Q_{1}, \ldots, Q_{n}$. The reality of all cyclic periods of $\mathrm{d} \xi(P)$ implies that the related residues are imaginary; as in Subsection 2.3 we denote them by $-i \alpha_{j}\left(\alpha_{j} \in \mathbb{R}\right)$. With $\left(a_{j}, b_{j}\right), j=1, \ldots, g$ the usual basis of homology cycles on $\mathcal{L}$, the cyclic periods of $\mathrm{d} \xi$ are given by

$$
\begin{aligned}
A_{j} & =\oint_{a_{j}} \mathrm{~d} \xi, \quad B_{j}=\oint_{b_{j}} \mathrm{~d} \xi, \quad j=1, \ldots, g, \\
2 \pi \alpha_{j} & =\oint_{C_{j}} \mathrm{~d} \xi, \quad j=1, \ldots, n,
\end{aligned}
$$

where $C_{j}$ are small contours enclosing the points $Q_{j}$. According to our assumption, all these periods are real; therefore the function $\rho(P)=\operatorname{Im} \xi(P)$ is single-valued on $\mathcal{L}$, and $x(P)=\operatorname{Re} \xi(P)$ has cyclic periods coinciding with (43).

Suppose now that $\mathcal{E}(x, \rho)$ is some solution of the Ernst equation. For $\mathcal{E}(x, \rho)$ to be single-valued on $\mathcal{L}$, the following periodicity conditions must be satisfied:

$$
\begin{aligned}
\mathcal{E}\left(x+A_{j}, \rho\right) & =\mathcal{E}\left(x+B_{j}, \rho\right)=\mathcal{E}(x, \rho), \quad j=1, \ldots, g, \\
\mathcal{E}\left(x+2 \pi \alpha_{j}, \rho\right) & =\mathcal{E}(x, \rho), \quad j=1, \ldots, n .
\end{aligned}
$$

Since it is impossible to construct a continuous non-constant function on $\mathbb{R}$ having two real periods $L_{1}$ and $L_{2}$ such that the ratio $L_{1} / L_{2}$ is irrational, we must require that all cyclic periods $A_{j}, B_{j}$ and $2 \pi \alpha_{j}$ satisfy 


$$
\begin{aligned}
& 2 \pi \alpha_{j}=k_{j} L, \quad j=1, \ldots, n, \\
& A_{j}=m_{j} L, \quad B_{j}=n_{j} L, \quad j=1, \ldots, g,
\end{aligned}
$$

for some $L \in \mathbb{R}$ and certain integers $k_{j}, m_{j}, n_{j}$. If $\mathcal{E}(x, \rho)$ is periodic with period $L$,

$$
\mathcal{E}(x+L, \rho)=\mathcal{E}(x, \rho),
$$

the function $\mathcal{E}(x(P), \rho(P))$ is single-valued on all of $\mathcal{L}$. The periodicity of the full metric (42) is ensured if the same periodicity constraints are obeyed by the functions $k$ and $A$, i.e.

$$
k(x+L, \rho)=k(x, \rho), \quad A(x+L, \rho)=A(x, \rho) .
$$

At least for the static case, these relations were established in the previous section. Now we recognize that it is precisely the periodicity constraints (44) and (45) which lead to the discretization of moduli space.

Physically we can distinguish two essentially different situations:

(i) For $k_{j}=m_{j}=n_{j}=0$, all cyclic periods of $\mathrm{d} \xi$ vanish, and $\mathrm{d} \xi(P)=\mathrm{d} h(P)$ for some meromorphic function $h(P)$ on $\mathcal{L}$; there are then no restrictions on the surface $\mathcal{L}$. The condition (46) becomes empty, and $\mathcal{E}(h, \bar{h})$ solves (5) without further ado. In particular, we can choose $\mathcal{E}(x, \rho)$ to be asymptotically flat (corresponding to the Schwarzschild solution, say); then the metric (42) will also be asymptotically flat near the poles of $\mathrm{d} \xi(P)=\mathrm{d} h(P)$ (which are of second order at least), where $x, \rho \rightarrow \infty$. The number of black holes on the symmetry axis $\rho=0$ coincides with the number of poles of $h(P)$.

(ii) If not all $k_{j}, m_{j}, n_{j}$ in (44), (45) vanish, the differential $\mathrm{d} \xi$ may in principle have poles of arbitrary order. For definiteness, let us restrict attention to the $L$-periodic Schwarzschild solution derived above. The symmetry axis $\rho=0$ now consists of several disconnected components. These may be either homeomorphic to $S^{1}$ or non-compact. The first possibility corresponds to the cross sections of $\mathcal{L}$ at "time" $\rho=0$. The second, on the other hand, is realized in the vicinity of any higher order pole of $\mathrm{d} \xi$, when the pole is approached in such a way that $\rho=0$ but $x \rightarrow \infty$. In the latter case, we end up with an infinite number of black holes in this region, which would have been asymptotically flat for $\mathcal{E}=1$. If one wants to avoid the occurrence of infinitely many black holes, one must consequently assume that $\mathrm{d} \xi$ has only simple poles (which is precisely the situation studied in string theory [4]). The neighbourhoods of the simple poles of $d \xi$ represent semi-infinite tubes, where $\rho \rightarrow \pm \infty$ (the sign coincides with the sign of the associated residue as we explained in Subsection 2.3). At the "tip" of any such tube, the metric (42) behaves like the Kasner solution. The number of black holes on each separate symmetry axis $\rho=0$ is equal to the cyclic period of $\mathrm{d} \xi$ along this contour.

In both cases, the metric (42) near any zero of $\mathrm{d} \xi$ describes a cosmic string just as for $\mathcal{E}=1$; the excess angles are given by $2 \pi k$, where $k$ is the order of the zero, and the various functions appearing in (42) are to be evaluated at this zero.

An artist's view of the section $\phi=$ const., $t=$ const., $\rho \geqslant 0$ of a typical manifold of the second type is given in Fig. 2. Analogous pictures for $\mathrm{d} \xi=\mathrm{d} h$ would look precisely 


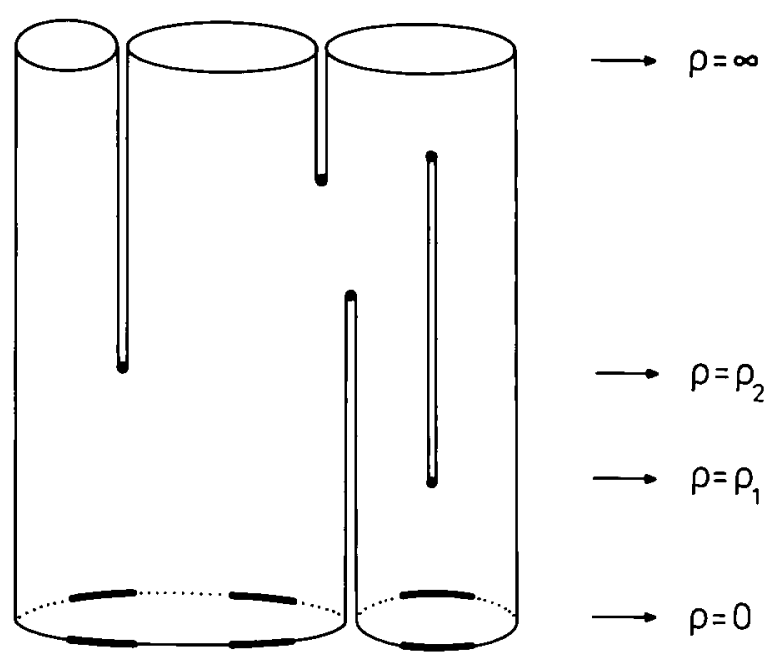

Fig. 2. Example of a static solution with two symmetry axes $(\rho=0)$ and three asymptotic regions $(\rho=\infty)$ for non-vanishing cyclic periods of $\mathrm{d} \xi$. The genus of $\mathcal{L}$ depends on how the surface is extended to negative $\rho$; for a reflection symmetric surface, it would be three. The solid points represent cosmic strings of radii $\rho=\rho_{1}, \rho_{2}, \ldots$, and the solid segments on the contours $\rho=0$ correspond to the horizons of the respective black holes.

like the usual diagrams representing a Riemann surface as a covering of the complex plane; the only difference is that here we get a covering of the upper half-plane only. The number of sheets coincides with the number of black holes and is equal to the number of poles of $h(P)$.

\section{Action of the Virasoro-Witt generators on the "partially discretized" moduli space}

We now wish to address the question of extra symmetries for the new solutions that we have constructed in this paper. Contrary to the axisymmetric solutions studied in the literature so far, our solutions depend on extra topological degrees of freedom, namely the moduli of the (punctured) Riemann surface $\mathcal{L}$. We will show that there is an analog of the solution generating symmetries acting on these topological degrees of freedom. Together with the original solution generating symmetries not affecting the topology of the world-sheet, they form a group which contains and extends the Geroch group.

To display the new symmetries, we recall that (as shown for instance in Ref. [29]) it is possible to define an action of a Virasoro-Witt algebra on the moduli space of algebraic curves of genus $g$. For all but finitely many generators, this action simply corresponds to a reparametrization of the coordinates, and hence has no physical significance. The remaining generators, on the other hand, correspond to genuine deformations of the conformal structure, and thus act non-trivially on the moduli space, yielding new solutions that cannot be "reached" by the Geroch group. We note that some action of 
a Virasoro-Witt algebra on the solutions of the Ernst equation (or, more precisely, on the spectral parameter plane) was already exhibited in Ref. [23] (cf. also Ref. [16]). However, this action does not give anything new beyond the action of the Geroch group itself. Rather, its action merely "stirs" the singularities of $\Psi$ in the $\lambda$-plane. In contrast, the non-trivial action of the Virasoro-Witt generators in our case is due to the presence of topological degrees of freedom on the world-sheet (we will ignore the torus $(g=1)$ as it presents no qualitatively new features).

Consider an arbitrary curve $\mathcal{L}$ of genus $g$ and choose some fixed point $P_{0}$ on it with related local parameter $\tau$ in a local neighborhood of $P_{0}$ (so that $\tau\left(P_{0}\right)=0$ ). On the boundary $\partial D$ of a small disc $D$ of radius $R$ around $P_{0}$, we define the vector fields

$$
t_{k}=-\tau^{k} \frac{\partial}{\partial \tau}, \quad k \in \mathbb{Z}
$$

Evidently, they generate a Virasoro-Witt algebra Vir. The space of these vector fields can be represented as a direct sum

$$
V=V_{-} \oplus V_{0} \oplus V_{+}
$$

Here, $V_{-}$is defined to consist of all vector fields admitting a holomorphic extension into the disc, i.e. the interior of the boundary $\partial D$; similarly, $V_{+}$consists of all vector fields possessing a holomorphic extension to the complement $\mathcal{L} \backslash D$ of the disc, i.e. the exterior of $\partial D$. The remaining vector fields, which can be extended holomorphically neither into $D$ nor into its complement, span the subspace $V_{0}$. Now it is known [29] that always

$$
\operatorname{dim}_{\mathbb{C}} V_{0}=3 g-3,
$$

and, if $P_{0}$ is not a Weierstrass point on $\mathcal{L}$, a basis in $V_{0}$ is given by

$$
t_{k}, k=-1, \ldots,-3 g-3 \text {. }
$$

We are now ready to define the action of an arbitrary vector field $\nu \in V_{0}$ on the moduli space by the following procedure [30,29]. Cut the curve $\mathcal{L}$ along $\partial D$; this gives two disconnected parts $D$ and its complement $\mathcal{L} \backslash D$, whose boundary points we label by $P_{+}$and $P_{-}$, respectively. We then shift the boundary points relative to one another after the replacement of $P_{+}$by $\exp (\beta v) P_{+}$(i.e. shifting $P_{+}$by the $S^{1}$ diffeomorphism generated by the vector field $v$ ), and then glue the pieces back together. In general, the conformal structure of the new curve $\mathcal{L}_{\beta}$ will be different. According to Ref. [31], the variation of the $b$-period matrix $B$ of $\mathcal{L}$ with respect to this transformation is described by the formula

$$
\left.\frac{\partial \boldsymbol{B}_{m n}}{\partial \beta}\right|_{\beta=0}=\oint_{\partial D} \mathrm{~d} U_{n} \mathrm{~d} U_{m} \nu,
$$

where $\mathrm{d} U_{n}$ (for $n=1, \ldots, g$ ) is a normalized basis of holomorphic differentials on $\mathcal{L}$ (the expression $\mathrm{d} U_{n} \mathrm{~d} U_{m} v$ is a one-form). 
Now we see that this variation is zero for $v \in V_{-}$and $v \in V_{+}$because the integration contour $\partial D$ can be deformed to a point if $v$ admits a holomorphic extension into $D$, or "pulled off the back of $\mathcal{L}$ ", if there is a holomorphic extension to the complement of $D$. Since the matrix of $b$-periods completely determines the conformal structure of $\mathcal{L}$, a deformation of the conformal structure requires $v \in V_{0}$. Since furthermore $\operatorname{dim}_{\mathbb{C}} V_{0}=$ $3 g-3$ precisely coincides with dimension of the moduli space of $\mathcal{L}$, we conclude that all generators of $V_{0}$ vary the conformal structure (observe that $V_{0}=V \ominus V_{-} \ominus V_{+}$is not a subalgebra of $V$, in contrast to $V_{-}$and $V_{+}$).

For the sake of clarity, let us first consider case where $\mathrm{d} \xi(P)=\mathrm{d} h(P)$ for some meromorphic function $h(P)$ on $\mathcal{L}$. We have to vary the set $(\mathcal{L}, h(P))$, i.e. the moduli of the Riemann surfaces of a given genus $g$ with $n$ punctures $R_{1}, \ldots, R_{n}$. In order to also vary the punctures, we must add $n$ further generators from $V_{+}$to $V_{0}$, which must be such that the matrix $s_{j}\left(R_{i}\right)$ is non-degenerate (although the value of the determinant of this matrix depends on the choice of local coordinates at the points $R_{j}$, the vanishing or non-vanishing of it is a coordinate-independent property) where the new generators are designated by $s_{j}(j=1, \ldots, n)$, and the related subspace of $V_{+}$by $V_{n} \subset V_{+}$. As a result, we can vary the set $\left(\mathcal{L}, R_{j}\right)$ by an arbitrary generator from

$$
V_{0} \oplus V_{n}=V \ominus V_{-} \ominus\left(V_{+} \ominus V_{n}\right) \text {. }
$$

Of course, this linear subpace of $V$ of dimension $3 g-3+n$ is not a subalgebra, in contrast to $V_{-}$and $V_{+}$. Generically, we can choose $s_{j}=t_{j}, j=-3 g-4, \ldots,-3 g-3-n$; then $V_{+} \ominus V_{n}$ is also a subalgebra and $V_{0} \oplus V_{n}$ is a "two-sided" coset space.

Next consider the more complicated situation, where $\mathrm{d} \xi(P)$ is a differential of the third kind on $\mathcal{L}$ subject to the discretization conditions (44), (45), and we have to vary the set of data $(\mathcal{L}, \mathrm{d} \xi)$. Denote the poles of $\mathrm{d} \xi$ by $Q_{1}, \ldots, Q_{n}$ and keep the related residues (discretized according to (44)) fixed. In analogy to the previous case, we have to add $n$ generators $s_{j} \in V_{+}$to $V_{0}$ in order to vary the points $Q_{j}$, such that the matrix $s_{j}\left(Q_{i}\right)$ is non-degenerate. Now, however, to preserve condition (45), we have to keep the periods $A_{j}, B_{j} j=1, \ldots, n$ fixed, since it is impossible to vary the integers $m_{j}$ and $n_{j}$ by an infinitesimal transformation. Assuming $A_{j}$ and $B_{j}$ fixed, consider the orthogonal decomposition

$$
V_{0} \oplus V_{n}=\tilde{V} \oplus \tilde{V}^{\perp},
$$

where the generators from $\tilde{V}$ do not vary $A_{j}$ and $B_{j}$. Obviously, since $A_{j}, B_{j} \in \mathbb{R}$, we have $\operatorname{dim}_{\mathbb{C}} \tilde{V}^{\perp}=g$ and

$$
\operatorname{dim}_{\mathbb{C}} \tilde{V}=3 g-3+n-g=2 g+n-3 \text {. }
$$

So the linear subspace

$$
\tilde{V}=V \ominus V_{-} \ominus\left(V_{+} \ominus V_{n}\right) \ominus \tilde{V}^{\perp}
$$

of the full Virasoro-Witt algebra corresponds to variations of the topological degrees of freedom of the world-sheet $\mathcal{L}$ in the second case.

The resulting symmetry algebra involving both propagating and topological degrees of freedom is the product of $V_{0} \oplus V_{n}$ and the Geroch algebra (i.e. $A_{1}^{(1)}$ ) if $\xi(P)$ is 
a meromorphic function on $\mathcal{L}$, and the product of the periodic analog of the Geroch algebra and the linear subspace $\tilde{V}$ otherwise.

\section{Concluding remarks}

There are several open questions that we have barely touched on and which merit further investigation. Amongst other things, it is necessary to clarify the structure of the space of periodic solutions of the Ernst equation and to investigate the global properties of the Geroch group acting on periodic solutions. This would permit the verification of our conjecture that there is a one-to-one correspondence between the asymptotically flat solutions of the Ernst equation and its asymptotically-Kasner periodic solutions, and perhaps to answer the question of whether there arise new and unphysical singularities in such solutions. Secondly, it would be desirable to understand in more detail the structure of the "discretized" moduli space and the action of the Virasoro-Witt generators on it.

Our use of string-inspired technology also suggests possible applications of our results in the context of string theory. A "stringy" interpretation of the metric (6) was already proposed in Ref. [16], where it was pointed out that the conformal factor (Liouville degree of freedom) $\sigma \equiv k-\frac{1}{2} \log (\mathcal{E}+\overrightarrow{\mathcal{E}})$ and the "dilaton" field $\rho$ together appear in the equations of motion in such a way that they can be interpreted as longitudinal target space degrees of freedom. All degrees of freedom can be combined into a Lorentzian "target space" metric

$$
\mathrm{d} s^{2}=\mathrm{d} \rho \mathrm{d} \sigma+\rho \frac{\mathrm{d} \mathcal{E} \mathrm{d} \overline{\mathcal{E}}}{(\mathcal{E}+\overline{\mathcal{E}})^{2}},
$$

such that the equations of motion (1) and (2) can be rederived from (49) by varying with respect to $\mathcal{E}$ and $\sigma$; the first order equation in (9) for the conformal factor and its complex conjugate are obtained as the "Virasoro conditions". Accordingly, the "matter" degrees of freedom residing in $\mathcal{E}$ (alias the transverse polarization states of the graviton) are reinterpreted as transverse target space degrees of freedom.

Of course, (49) does not correspond to a conformally invariant theory because of its explicit dependence on the conformal factor $\sigma$. However, in Liouville theory as well, the conformal factor does not decouple, but conformal invariance is nevertheless restored at the quantum level as the Liouville degree of freedom "adjusts" its contribution to the conformal anomaly in the required manner (see e.g. Ref. [32] for reviews). Although the viability of this interpretation of (49) remains to be tested, we feel encouraged by the result that the sigma model based on (49) is one-loop finite even though the target space is not Ricci-flat [24] (see also Ref. [33]).

A similar proposal to apply the Ernst equation to the description of four-dimensional string backgrounds was recently made in Ref. [34], where the target space is taken to be an axisymmetric stationary four-manifold. The string zero-modes propagating on this background are an antisymmetric tensor $B_{\mu \nu}$ and a dilaton (not to be confused with $\rho$ above), whose interactions are governed (after dualization of $B_{\mu \nu}$ ) by an $S L(2, \mathbb{R})$ sigma model. In the axisymmetric reduction, the Geroch group is replaced by the affine Kac-Moody extension of $O(2,2) \cong S L(2, \mathbb{R}) \times S L(2, \mathbb{R})$ in the usual way; it can 
be interpreted as a group acting on the space of string backgrounds [34]. The results obtained here suggest that the existence of even bigger symmetry groups on the space of string backgrounds which also act on the moduli of the world-sheet and possibly mix them with the propagating degrees of freedom.

\section{Acknowledgement}

D.K. thanks Yavus Nutku for important discussions.

\section{References}

[1] F. Ernst, Phys. Rev. 167 (1968) 1175.

[2] C. Hoenselars and W. Dietz (eds.), Exact solutions of Einstein's Equations: Techniques and Results, Lecture Notes in Physics 205, Springer Verlag, 1984.

[3] S. Mandelstam, Nucl. Phys. B 64 (1973) 205.

[4] S. Giddings and S. Wolpert, Commun. Math. Phys. 109 (1987) 177.

[5] R. Geroch, J. Math. Phys. 12 (1971) 918; 13 (1972) 394.

[6] V.A. Belinskii and V.E. Zakharov, Sov. Phys. JETP 48 (1978) 985

[7] D. Maison, Phys. Rev. Lett. 41 (1978) 521.

[8] F. Ernst and I. Hauser, Phys. Rev. D 20 (1979) 362.

[9] Y. Alexeev, Trudy Mat. Inst. Steklov 176 (1987) 211.

[10] G. Neugebauer, J. Phys. A 12 (1979) L67.

[11] D. Korotkin, Theor. Math. Phys. 77 (1989) 1018;

D. Korotkin and V. Matveev, Leningrad Math. J. 1 (1990) 379.

[12] A.P. Veselov, Theor. and Math. Physics, 54 (1983) 155.

[13] G. Neugebauer and D. Kramer, in Ref. [2].

[14] D. Korotkin, Class. Quant. Gravity 10 (1993) 2587.

[15] R. Griffiths, Colliding Plane Waves in General Relativity, Cambridge University Press (1991).

[16] H. Nicolai, in: Recent Aspects of Quantum Fields, Proceedings, Schladming 1991, eds. H. Mitter and H. Gausterer, Springer Verlag, 1991.

[17] J. Ehlers, Dissertation, University of Hamburg (1957);

R. Matzner and C. Misner, Phys. Rev. 154 (1967) 1229;

W. Kinnersley and D. Chitre, J. Math. Phys. 18 (1977) 1538; 19 (1978) 1926;

B.K. Harrison, Phys. Rev. Lett. 41 (1978) 1197;

C. Cosgrove, J. Math. Phys. 21 (1980) 2417;

K. Ueno and Y. Nakamura, Phys. Lett. B 117 (1982) 208.

[18] E. Cremmer, S. Ferrara and J. Scherk, Phys. Lett. B 74 (1978) 61;

E. Cremmer and B. Julia, Nucl. Phys. B 159 (1979) 141.

[19] B. Julia, in: Superspace and Supergravity, eds. S.W. Hawking and M. Rocek, Cambridge University Press (1980).

[20] P. Breitenlohner and D. Maison, Ann. Inst. Poincaré 46 (1987) 215;

P. Breitenlohner, D. Maison and G.W. Gibbons, Commun. Math. Phys. 120 (1988) 295.

[21] B. Julia, in: Unified Theories and Beyond, Proc. 5th Johns Hopkins Workshop on Current Problems in Particle Theory, Johns Hopkins University, Baltimore, 1982; in: Vertex Operators in Mathematics and Physics, eds. J. Lepowsky, S. Mandelstam and I. Singer, Springer Verlag, Heidelberg, 1984.

[22] H. Nicolai, Nucl. Phys. B 414 (1994) 299.

[23] D. Maison, Geroch Group and Inverse Scattering Method, preprint MPI-PAE/PTh 80/88 (1988);

B. Hou and W. Lee, Lett. Math. Phys. 13 (1987) 1.

[24] B. de Wit, M. Grisaru, E. Rabinovici and H. Nicolai, Phys. Lett. B 286 (1992) 78.

[25] A. Vilenkin, Phys. Rep. 121 (1985) 263. 
[26] E. Witten, Commun. Math. Phys. 121 (1989) 351.

[27] R.C. Myers, Phys. Rev. D 35 (1987) 455.

[28] D. Kramer et al., Exact solutions of Einstein field equation; Cambridge University Press, 1980.

[29] M. Kontsevich, Funct. Anal. and its Appl. 21 (1987) 78.

[30] P. Grinevich and A. Orlov, Funct. Anal. and its Appl. 24 (1990) 72.

[31] M. Schiffer and D.K. Spencer, Functionals on Finite Riemann Surfaces, Princeton University Press, 1954.

[32] E. D'Hoker, Mod. Phys. Lett. A 6 (1991) 745;

L. Alvarez-Gaumé and C. Gomez, Topics in Liouville Theory, preprint CERN-TH 6175 (1991).

[33] A.A. Tseytlin, Int. J. Mod. Phys. D I (1992) 223.

[34] 1. Bakas, $O(2,2)$-Transformations and the String Geroch Group, preprint hep-th/9402016. 